\title{
Long non-coding RNA NEAT1 participates in ventilator-induced lung injury by regulating miR-20b expression
}

\author{
YI LIU, GANG TANG and JINYU LI \\ Department of Anesthesiology, The Affiliated Huaian No.1 People's Hospital of \\ Nanjing Medical University, Huai'an, Jiangsu 211100, P.R. China
}

Received May 14, 2021; Accepted August 11, 2021

DOI: $10.3892 / \mathrm{mmr} .2022 .12582$

\begin{abstract}
Long non-coding (lnc)RNA nuclear enriched abundant transcript 1 (NEAT1) has been reported to serve an important role in cancer, but its effects on ventilator-induced lung injury (VILI) remain unclear. The present study aimed to investigate the role of IncRNA NEAT1 in alveolar macrophages (AMs) on ventilator-induced lung injury (VILI). Mouse and cell models were established to detect NEAT1 expression, pathological changes in lung tissues, apoptosis of AMs, expression of the M1 phenotype marker, CD86 and M2 phenotype marker, CD206, and the expression levels of interleukin (IL)-1 $\beta$, IL-6, tumor necrosis factor (TNF)- $\alpha$ and inducible nitric oxide synthase (iNOS). The associations between NEAT1, microRNA (miRNA/miR)-20b and STAT3 were predicted using StarBase and TargetScan, and verified via the dual-luciferase reporter and RIP assays. NEAT1 short hairpin RNA and miR-20b inhibitor were co-transfected into AMs to assess the effect of NEAT1 and miR-20b in VILI. The results demonstrated that NEAT1 was highly expressed in lung tissues of VILI mice and cell stretch (CS) treated AMs. Furthermore, NEAT1 knockdown inhibited lung injury and cell apoptosis induced by VILI. Compared with VILI mice or CS-treated AMs, NEAT1 knockdown accelerated the phenotypic transformation from M1 to M2, and decreased the expression levels of IL-1 $\beta$, IL- 6 , TNF- $\alpha$ and iNOS. Notably, miR-20b was identified as the target of NEAT1, and STAT3 was the target of miR-20b. NEAT1 knockdown decreased STAT3 protein expression, the effects of which were reversed following transfection with miR-20b inhibitor. Furthermore, the protective effect of NEAT1 knockdown on VILI was reversed following transfection with miR-20b inhibitor. Taken
\end{abstract}

Correspondence to: Dr Jinyu Li, Department of Anesthesiology, The Affiliated Huaian No.1 People's Hospital of Nanjing Medical University, 1 Huanghe West Road, Huaiyin, Huai'an, Jiangsu 211100, P.R. China

E-mail: jinyuli3568@163.com

Key words: long non-coding RNA nuclear enriched abundant transcript 1, ventilator-induced lung injury, alveolar macrophages, phenotypic transformation, microRNA-20b together, the results of the present study suggest that NEAT1 knockdown promotes phenotypic transformation of AMs from M1 to M2 and alleviates lung injury and apoptosis of VILI by regulating miR-20b expression.

\section{Introduction}

Ventilator-induced lung injury (VILI) is caused by the interaction between the mode in which the ventilator delivers to the lung parenchyma and the mode in which the lung parenchyma accepts (1). VILI is the most serious complication of mechanical ventilation (MV) and increases the morbidity and mortality of patients receiving ventilator therapy (1). Currently, the main measures to alleviate VILI include high frequency oscillatory ventilation, low tidal volume and inspiratory pressure ventilation, prone position ventilation and positive end-expiratory pressure (2). Although several studies have focused on VILI (3-6), the effectiveness of current treatment regimens is still limited. Thus, it is important to identify more effective therapeutic targets to alleviate the pain of patients with VILI.

Aggregation of inflammatory cells and overproduction of inflammatory cytokines are two key processes in the pathogenesis of VILI $(7,8)$. Cytokines released by pro-inflammatory factors can increase the permeability of the alveolar capillary barrier, resulting in pulmonary dysfunction (9). Macrophages are the most abundant immune cells in several organs, including the lungs, and play an important role in restoring tissue homeostasis after triggering inflammatory signals $(10,11)$. Previous studies have reported that macrophages effectively respond to environmental signals by changing their phenotype, thus altering their physiological effect to immune responses $(12,13)$. Macrophages are classified as M1 and M2 phenotypes (14), according to their immunomodulatory function. M1 macrophages (classically activated) can enhance inflammatory response, whereas M2 macrophages (alternatively activated) reduce inflammatory response and promote tissue regeneration $(15,16)$. Transition of macrophages can occur between these two phenotypes under certain conditions $(17,18)$. Safavian et al (13) demonstrated that a reduction in the M2 phenotype of alveolar macrophages (AMs) increased lipopolysaccharide (LPS)-induced lung injury. Therefore, the phenotypic transformation of AMs may inhibit the progression of VILI. 
Increasing evidence suggest that long non-coding RNAs (lncRNAs) act as competing endogenous RNAs (ceRNAs) and interact with microRNAs (miRNAs) to regulate gene expression (19-21). Previous research has indicated that MALAT1 knockdown alleviates neuronal cell death by upregulating miR-30a expression in cerebral ischemic stroke (22). Wang et al (23) demonstrated that GAS5 knockdown inhibits renal tubular epithelial fibrosis by competitively binding to miR-96-5p. Notably, nuclear enriched abundant transcript 1 (NEAT1) knockdown inhibits the fibrogenesis and epithelial-to-mesenchymal transition in diabetic nephropathy (DN) by targeting miR-27b-3p and ZEB1 expression (24). Zhou et al (25) reported that NEAT1 expression is upregulated in LPS-induced A549 cells, and inhibition of lncRNA NEAT1 inhibits the production of inflammatory cytokines. In hepatic ischemia/reperfusion (I/R) injury, lncRNA HOTAIR also regulates autophagy via the miR-20b-5p/ATG7 axis (26). However, whether lncRNA NEAT1 in AMs participates in the VILI process by regulating miR-20b expression remains unclear.

The present study aimed to investigate the effect of lncRNA NEAT1 in AMs on VILI. For this purpose, mouse and cell models of VILI were established to detect the effect of NEAT1 on pathological changes, apoptosis and levels of inflammatory markers. Furthermore, the downstream miRNA and gene of NEAT1 were predicted to explore the underlying molecular mechanism of NEAT1 in VILI.

\section{Materials and methods}

VILI mouse model. A total of 40 adult male C57BL/6 mice (age, $10 \pm 2$ weeks; weight, $25 \pm 2 \mathrm{~g}$ ) were purchased from Nanjing Biomedical Research Institute of Nanjing University. The animals were fed with common feed and tap water in a specific pathogen-free laboratory with a $12 \mathrm{~h}$ light/dark cycle at $22 \pm 1^{\circ} \mathrm{C}$ and $45-55 \%$ relative humidity. The mice were randomly divided into three groups ( $\mathrm{n}=8 /$ group), as follows: i) Control (mice without any treatment); ii) Sham (mice that underwent tracheotomy but were still breathing spontaneously); and iii) VILI (mice that underwent tracheotomy and MV for $4 \mathrm{~h}$ ) groups. To construct the VILI mouse model, mice were anaesthetized with ketamine $(100 \mathrm{mg} / \mathrm{kg})$ and xylazine $(10 \mathrm{mg} / \mathrm{kg}$ ) by intraperitoneal injection and tracheotomy was performed. The ventilator (Harvard Apparatus) was connected to the mice and the animals were ventilated with $30 \mathrm{ml} / \mathrm{kg}$ air at a rate of 70 breaths $/ \mathrm{min}$ for $4 \mathrm{~h}$.

RNA interference. NEAT1 short hairpin (sh)RNA (sh-NEAT1, 5'-GCTTATAAGTTTGTTGTGTTG-3') and negative control (sh-NC, 5'-GGTTCGGTTTATTGAGTTTAT-3') were purchased from Shanghai GenePharma Co., Ltd. The second-generation lentivirus encoding green fluorescent protein (GFP)-expressing NEAT1 shRNA (GFP-NEAT1 lentivirus) was generated for the present study. The pCMVdr-8.91 and pMD2G plasmids (Thermo Fisher Scientific, Inc.) were used as package systems. The recombinant lentivirus was generated by co-transfection of 293FT cells (Thermo Fisher Scientific, Inc.) with lentiCRISPR V2 (10 $\mu \mathrm{g}$; Addgene, Inc.), pCMVdr-8.91 $(7.5 \mu \mathrm{g})$, and pMD2G plasmids $(5 \mu \mathrm{g})$, using Lipofectamine ${ }^{\circledR} 3000$ reagent (Invitrogen; Thermo Fisher
Scientific,Inc.) at room temperature. The medium was collected and ultracentrifuged at $12,000 \times \mathrm{g}$ for $3 \mathrm{~h}$ at $4^{\circ} \mathrm{C}$ and filtered using $0.22-\mu \mathrm{m}$ filters, $48 \mathrm{~h}$ post-transfection. The resulting pellets were resuspended in PBS, cooled and stored at $-80^{\circ} \mathrm{C}$. For transduction of target cells, the lentivirus vector (MOI=20) was directly added to the complete medium, followed by transfection at $37^{\circ} \mathrm{C}$ for $72 \mathrm{~h}$. Stably transfected cells were selected by adding puromycin $(6 \mathrm{~g} / \mathrm{ml})$ in medium and the maintenance concentration of puromycin in transfected cells was $3 \mathrm{~g} / \mathrm{ml}$. The infection efficiency of AMs was observed under a fluorescence microscope (magnification, x100). Subsequent experiments were performed $72 \mathrm{~h}$ post-transfection.

A total of 16 mice were randomly divided into two groups (eight mice/group), VILI + sh-NC and VILI + sh-NEAT1 groups. Mice in the VILI + sh-NEAT1 group received sh-NEAT1 by intravenous injection prior to VILI treatment, while mice in the VILI + sh-NC group received sh-NC by intravenous injection prior to VILI treatment. All mice were sacrificed via cervical dislocation after anesthesia with $50 \mathrm{mg} / \mathrm{kg}$ pentobarbital sodium. All animal experiments were approved by the Ethics Committee of The Affiliated Huaian No.1 People's Hospital of Nanjing Medical University (Huaian, China; approval no. HASDY20201009).

Sample collection. After establishing the VILI model or performing spontaneous respiration, the mice were sacrificed by intraperitoneal injection of $100 \mathrm{mg} / \mathrm{kg}$ sodium pentobarbital. The right lung was collected for bronchoalveolar lavage fluid (BALF) collection, and the left lung was collected for histopathology and reverse transcription-quantitative PCR (RT-qPCR) detection of NEAT1. BALF and the lung tissues were collected and stored at $-80^{\circ} \mathrm{C}$ until subsequent experimentation. The total protein concentration of $\mathrm{BALF}$ was determined using the BCA method (Beyotime Institute of Biotechnology).

Histopathology. Lung tissues were fixed in $10 \%$ formaldehyde at $4^{\circ} \mathrm{C}$ overnight and embedded in paraffin. Paraffin-embedded tissue samples were cut into 3- $\mu$ m-thick sections for hematoxylin and eosin (H\&E) staining. Samples were subsequently stained with hematoxylin for $10 \mathrm{~min}$ and eosin for $10 \mathrm{sec}$ at room temperature. The sections were observed under a light microscope (magnification, $\mathrm{x} 200$ ).

ELISA. The levels of interleukin (IL)-1 $\beta$ (cat. no. SEA563Mu; Wuhan USCN Business Co., Ltd.), IL-6 (cat. no. SEA079Mu; Wuhan USCN Business Co., Ltd.), tumor necrosis factor- $\alpha$ (TNF- $\alpha$; cat. no. SCA133Mu; Wuhan USCN Business Co., Ltd.) and inducible nitric oxide synthase (iNOS; cat. no. ml057773; Shanghai Enzyme-linked Biotechnology Co., Ltd.) in BALF and AMs were determined using respective ELISA kits (Thermo Fisher Scientific, Inc.), according to the manufacturer's instructions.

Isolation, collection and counting of AMs. BALF was collected by injecting PBS intratracheally three times. BALF was centrifuged at $500 \mathrm{x}$ g for $10 \mathrm{~min}$ at $4^{\circ} \mathrm{C}$ and the supernatant was discarded. The red blood cells were lysed using ACK lysis buffer (Beyotime Institute of Biotechnology) for $5 \mathrm{~min}$. This was followed by centrifugation at $500 \mathrm{x} \mathrm{g}$ for 
$10 \mathrm{~min}$ at $4^{\circ} \mathrm{C}$, after which the supernatant was discarded and the pelleted cells were resuspended in RPMI-1640 medium (Gibco; Thermo Fisher Scientific, Inc.). The single cell suspension made of the culture solution was incubated overnight at $37^{\circ} \mathrm{C}$, the adherent cells were macrophages. A hemocytometer was used to count the number of AMs.

In vitro cell stretch $(C S)$ experiments. In vitro $\mathrm{CS}$ experiments were performed using the FX-5000T Flexercell Tension Plus system (Flexcell International Corp.) to stretch isolated AMs and simulate the stretching of MV. Briefly, AMs were loaded in a Flexercell FX-4000T strain unit; the cells were stretched for $4 \mathrm{~h}$ at a frequency of 30 cycles $/ \mathrm{min}(0.5 \mathrm{~Hz})$ and a $20 \%$ range with a stretch-to-relaxation ratio of 1:1 (27). The control cells were also placed in the Flexercell FX-4000T strain unit without mechanical stretching.

Cell transfection. Sh-NEAT1 and sh-NC, miR-20b mimics (50 nM; forward, 5'-ACUGCAGUGUGAGCACUUCUAG-3' and reverse, 5'-AGAAGUGCUCACACUGCAGUUU-3') and mimics NC (50 nM; forward, 5'-UUCUCCGAACGUGUCACG UTT-3' and reverse, 5'-ACGUGACACGUUCGGAGAATT-3'), miR-20b inhibitor (100 nM; 5'-GTGCTCATAGTGCAGGTA GTT-3') and inhibitor NC (100 nM; 5'-GGGTCTGACGAG GTACTATTT-3'), STAT3 overexpression plasmid pcDNA3.1 (pc-STAT3, $5 \mu \mathrm{g}$ ) and the parental negative control (pc-NC, $5 \mu \mathrm{g}$ ) were all purchased from Shanghai GenePharma, Co., Ltd., and transfected into AMs using Lipofectamine ${ }^{\circledR} 3000$ reagent (Invitrogen; Thermo Fisher Scientific, Inc.) at $37^{\circ} \mathrm{C}$ for $48 \mathrm{~h}$, according to the manufacturer's instructions. Cells were harvested for subsequent experimentation $48 \mathrm{~h}$ post-transfection.

3-(4,5-dimethylthiazol-2-yl)-2,5-diphenyltetrazolium bromide (MTT) assay. The cell viability of AMs was detected using an MTT assay kit (Nanjing KeyGen Biotech Co., Ltd.). Cells $\left(1 \times 10^{4} /\right.$ well) were cultured in a 96 -well plate at $37^{\circ} \mathrm{C}$ for $48 \mathrm{~h}$. The MTT solution was added and incubated for $2 \mathrm{~h}$ at $37^{\circ} \mathrm{C}$. Following incubation, the supernatant was removed carefully and the insoluble formazan was dissolved in $150 \mu 1$ DMSO. The absorbance was measured at $570 \mathrm{~nm}$ to assess cell viability.

Apoptosis analysis. Apoptosis of AMs was detected using the Annexin V-FITC/propidium iodide (PI) apoptosis detection kit (Vazyme Biotech Co., Ltd.). Briefly, AMs were resuspended in $500 \mu \mathrm{l}$ binding buffer (Vazyme Biotech Co., Ltd.) at a density of $1 \times 10^{5}$ cells $/ \mathrm{ml}$. AMs were subsequently stained with Annexin V and PI for $10 \mathrm{~min}$ at room temperature, in the dark. Apoptotic cells were detected using a BD Accuri C6 Plus flow cytometer (BD Biosciences) and FACSDiva software (version 6.13; BD Biosciences).

$R T-q P C R$. Total RNA was extracted from lung tissues and AMs using TRIzol ${ }^{\circledR}$ reagent (Invitrogen; Thermo Fisher Scientific, Inc.) and reverse transcribed into cDNA using the TaqMan MicroRNA Reverse Transcription kit (Thermo Fisher Scientific, Inc.) and PrimeScript RT Master Mix (Takara Biotechnology, Co., Ltd.). The cDNA synthesis was performed at $37^{\circ} \mathrm{C}$ for $15 \mathrm{~min}$ and $85^{\circ} \mathrm{C}$ for $5 \mathrm{sec}$. qPCR was subsequently performed using TaqMan $2 \mathrm{X}$ Universal PCR Master Mix (Thermo Fisher Scientific, Inc.). The following thermocycling conditions were used for qPCR: Initial denaturation at $95^{\circ} \mathrm{C}$ for $10 \mathrm{~min} ; 40$ cycles of $95^{\circ} \mathrm{C}$ for $1 \mathrm{~min}, 63^{\circ} \mathrm{C}$ for $2 \mathrm{~min}, 72^{\circ} \mathrm{C}$ for $1 \mathrm{~min}$; final $72^{\circ} \mathrm{C}$ for $10 \mathrm{~min}$. The following primer sequences were used for qPCR: NEAT1 forward, 5'-TGGCTAGCTCAGGGCTTCAG-3' and reverse, 5'-TCTCCT TGCCAAGCTTCCTTC-3'; STAT3 forward, 5'-CCTTCCTCA CCGTGTACTGG-3' and reverse, 5'-AGCGTAGGGTAAGGT TCTTGC-3'; CD68 forward, 5'-GCTACATGGCGGTGGAGTA CAA-3' and reverse, 5'-ATGATGAGAGGCAGCAAGATGG-3'; CD206 forward, 5'-TTCGACACCCATCGGAATT-3' and reverse, 5'-CACAAGCGCTGCGTGGAT-3'; GAPDH forward, 5'-AGTCAGCTCTCTCCTTTCAGG-3' and reverse, 5'-TCC ACCACCCTGTTGCTGTA-3'; miR-20b forward, 5'-GCTCAT AGTGCAGGTAGAA-3' and reverse, 5'-TGTCAACGATAC GCTACG-3'; and U6 forward, 5'-CTCGCTTCGGCAGCACA-3' and reverse, 5'-AACGCTTCACGAATTTGCGT-3'. Relative expression levels were calculated using the $2^{-\Delta \Delta \mathrm{Cq}}$ method (28) and normalized to U6 or GAPDH.

Dual-luciferase reporter assay. The dual-luciferase reporter assay was performed to validate the binding site between NEAT1 and miR-20b, and between miR-20b and STAT3, in AMs. The luciferase reporter plasmids (pGL3 vectors; Promega Corporation) containing NEAT1-wild-type (WT), NEAT1-mutant (MUT), STAT3-WT and STAT3-MUT were synthesized by Shanghai GenePharma Co., Ltd. The constructed plasmids were co-transfected into AM cells with miR-20b mimics or mimics NC using Lipofectamine ${ }^{\circledR} 3000$ (Invitrogen; Thermo Fisher Scientific, Inc.). After transfection for $48 \mathrm{~h}$, relative luciferase activities were detected using the Dual-Luciferase Reporter Assay kit (Promega Corporation). Relative luciferase activity was normalized to Renilla luciferase activity.

RNA immunoprecipitation (RIP) assay. The Magna RNA-binding protein immunoprecipitation kit (Millipore, Sigma) was used to perform the RIP assay, according to the manufacturer's instructions. Briefly, cells $\left(1 \times 10^{7}\right)$ were centrifugated at $3,000 \times \mathrm{g}$ at $4^{\circ} \mathrm{C}$ for $10 \mathrm{~min}$ and resuspended with RIPA buffer (100 $\mu$; Sigma-Aldrich; Merck KGaA) containing protease and RNase inhibitors. Cell lysates (100 $\mu \mathrm{l})$ were incubated with human anti-Ago 2 antibody (5 $\mu \mathrm{g}$; Cell Signaling Technology, Inc.) or negative control IgG (5 $\mu \mathrm{g}$; ProteinTech Group, Inc.) coated magnetic beads (50 $\mu \mathrm{l}$; Thermo Fisher Scientific, Inc.) at $4^{\circ} \mathrm{C}$ overnight, while shaking to aid digestion of proteins and the precipitated RNA was isolated. Beads were washed twice using PBS buffer (Sangon Biotech Co., Ltd.), and the mixture was centrifuged at $2,500 \mathrm{x} \mathrm{g}$ for $10 \mathrm{~min}$ at $4^{\circ} \mathrm{C}$. RNA was treated with proteinase $\mathrm{K}$ for $30 \mathrm{~min}$ at $55^{\circ} \mathrm{C}$ and extracted using TRIzol (Thermo Fisher Scientific, Inc.). The relative expression levels of NEAT1, miR-20b and STAT3 were determined via RT-qPCR analysis.

Western blotting. Total protein was extracted from lung tissues or AMs using RIPA lysate containing protease inhibitors (Thermo Fisher Scientific, Inc.). The enhanced BCA protein assay kit (Beyotime Institute of Biotechnology) was used to quantify the protein concentration. Protein $(50 \mu \mathrm{g} / \mathrm{per}$ lane) were separated via $10 \%$ SDS-PAGE, transferred onto PVDF membranes and blocked with non-fat milk for $1 \mathrm{~h}$ at room temperature. The membranes were incubated with primary 
antibodies against STAT3 (cat. no. ab68153; 1:1,000; Abcam) and GAPDH (cat. no. ab8245; 1:1,000; Abcam) overnight at $4^{\circ} \mathrm{C}$. Following the primary incubation, membranes were incubated with HRP-conjugated anti-rabbit IgG secondary antibody (cat. no. ab6721; 1:5,000; Abcam) at room temperature for $1 \mathrm{~h}$. Protein bands were visualized with ECL detection reagents (Cytiva), and blots were semi-quantitated using ImageJ software (version V1.8.0; National Institutes of Health).

Bioinformatics analysis. The DIANA tools database (http:// carolina.imis.athena-innovation.gr/diana_tools/web/index.php?r= lncbasev2\%2Findex-predicted) and differentially expressed genes (DEGs; GEO accession no. GSE148649; https://www. ncbi.nlm.nih.gov/geo/query/acc.cgi?acc=GSE148649) were used to screen the targets of IncRNA NEAT1. The binding sites between IncRNA NEAT1 and miR-20b were predicted using StarBase3.0 (http://starbase.sysu.edu.cn/index.php), while the binding sites between miR-20b and STAT3 were predicted using TargetScan 7.2 (http://www.targetscan.org/vert_72).

Statistical analysis. Statistical analysis was performed using SPSS 23.0 software (IBM Corp.) and GraphPad Prism 7.0 software (GraphPad Software, Inc.). All experiments were repeated three times and data are presented as the mean \pm SD. The comparisons among multiple groups were analyzed using one-way ANOVA followed by Tukey's post hoc test. The comparisons between two groups were analyzed using an unpaired Student's t-test. $\mathrm{P}<0.05$ was considered to indicate a statistically significant difference.

\section{Results}

NEATI knockdown alleviates lung injury caused by VILI. Abnormal NEAT1 expression was observed in VILI mice. The results demonstrated that NEAT1 expression was significantly higher in the lung tissues and BALF of the VILI group compared with the Sham group $(\mathrm{P}<0.001$; Fig. 1A and B). Furthermore, total protein in the BALF was significantly higher in the VILI group compared with the Sham group $(\mathrm{P}<0.001$; Fig. 1C).

To investigate the role of NEAT1 in VILI, sh-NEAT1 was transfected into mice prior to VILI treatment. H\&E staining demonstrated that VILI destroyed lung injury of mice, and NEAT1 knockdown suppressed the destructive effect of VILI on lung tissues (Fig. 1D). RT-qPCR analysis demonstrated that NEAT1 expression decreased in the VILI + sh-NEAT1 group compared with the VILI + sh-NC group $(\mathrm{P}<0.001$; Fig. 1E).

The activation of AMs is classified as classically activated M1 and alternatively activated M2 $(15,16)$. RT-qPCR analysis was performed to detect the mRNA expression levels of the M1 phenotype marker, CD68 and M2 phenotype marker, CD206. The results demonstrated that VILI increased CD68 mRNA expression but decreased CD206 mRNA expression, the effects of which were reversed following NEAT1 knockdown $(\mathrm{P}<0.001$; Fig. $1 \mathrm{~F}$ and $\mathrm{G})$. The ELISA assay was performed to assess the effect of NEAT1 on the expression levels of IL-1 $\beta$, IL-6, TNF- $\alpha$ and iNOS. The results demonstrated that NEAT1 knockdown decreased the expression levels of IL-1 $\beta$, IL-6, TNF- $\alpha$ and iNOS, the effects of which were reversed following VILI treatment $(\mathrm{P}<0.001$; Fig. $1 \mathrm{H})$.
NEAT1 knockdown decreases the cell injury of CS treatment on AMs. NEAT1 shRNA was transfected into AMs to detect the effect of NEAT1 in VILI in vitro. NEAT1 expression significantly decreased following transfection with NEAT1 shRNA ( $\mathrm{P}<0.001 ;$ Fig. 2A). CS experiments were performed to imitate the stretching of MV. Similar to the results of the VILI mouse model, CS significantly increased NEAT1 expression $(\mathrm{P}<0.001$; Fig. 2B), inhibited cell viability $(\mathrm{P}<0.05$; Fig. $2 \mathrm{C})$ and induced the apoptosis of AMs $(\mathrm{P}<0.001$; Fig. 2D). Notably, CS treatment increased CD68 mRNA expression ( $\mathrm{P}<0.001$; Fig. 2E) and decreased CD206 mRNA expression in AMs $(\mathrm{P}<0.001$; Fig. 2F) compared with the control group. The effect of NEAT1 on inflammation was assessed via the ELISA assay. The results demonstrated that CS treatment significantly increased the expression levels of IL-1 $\beta$, IL-6, TNF- $\alpha$ and iNOS in AMs compared with the control group (all $\mathrm{P}<0.001$; Fig. 2G).

NEAT1 shRNA was transfected into AMs to confirm the effect of NEAT1 on VILI. As presented in Fig. 2A and B, NEAT1 expression significantly decreased following transfection with NEAT1 shRNA $(\mathrm{P}<0.001)$. Notably, NEAT1 knockdown significantly increased cell viability $(\mathrm{P}<0.05$; Fig. 2C), inhibited cell apoptosis ( $\mathrm{P}<0.001$; Fig. 2D), decreased CD68 mRNA expression ( $\mathrm{P}<0.001$; Fig. 2E), increased CD206 mRNA expression $(\mathrm{P}<0.001$; Fig. $2 \mathrm{~F})$ and decreased the expression levels of IL-1 $\beta$, IL-6, TNF- $\alpha$ and iNOS in AMs (P<0.001; Fig. 2G).

NEAT1 sponges miR-20b expression in AMs. The underlying molecular mechanism of NEAT1 in VILI was further investigated. A total of 10 miRNAs were predicted to directly interact with NEAT1 via bioinformatics analysis (Fig. 3A). RT-qPCR analysis was performed to detect the expression levels of the miRNAs. The results demonstrated that the expression levels of the screened miRNAs increased following transfection with sh-NEAT1 in AMs, and the most significant increase was observed in miR-20b ( $\mathrm{P}<0.001$; Fig. 3B). The StarBase 3.0 database was used to predict the potential binding site between NEAT1 and miR-20b (Fig. 3C), and the dual-luciferase reporter assay was performed to confirm the interaction between NEAT1 and miR-20b. The results demonstrated that luciferase activity significantly decreased in cells co-transfected with NEAT1-WT and miR-20b mimic $(\mathrm{P}<0.01)$, while no significant changes were observed in cells co-transfected with NEAT1-MUT and miR-20b mimics (Fig. 3D). The results of the RIP assay proved that both NEAT1 and miR-20b were immunoprecipitated by anti-Ago2 ( $\mathrm{P}<0.001$; Fig. 3E). In addition, RT-qPCR analysis demonstrated that NEAT1 knockdown in both VILI mice $(\mathrm{P}<0.001$; Fig. 3F) and CS-treated AMs ( $<<0.001$; Fig. 3G) increased miR-20b expression compared with the VILI + sh-NC or CS + sh-NC groups. As expected, miR-20b expression significantly increased following transfection with miR-20b mimics and significantly decreased following transfection with miR-20b inhibitor (both $\mathrm{P}<0.001$; Fig. $3 \mathrm{H}$ ).

NEAT1 modulates miR-20b expression to regulate STAT3 in $A M s$. To determine the regulatory mechanism of miR-20b in VILI, the potential target genes of miR-20b were predicted using TargetScan. The results revealed that STAT3 has a binding site for miR-20b (Fig. 4A). The results of the dual-luciferase 

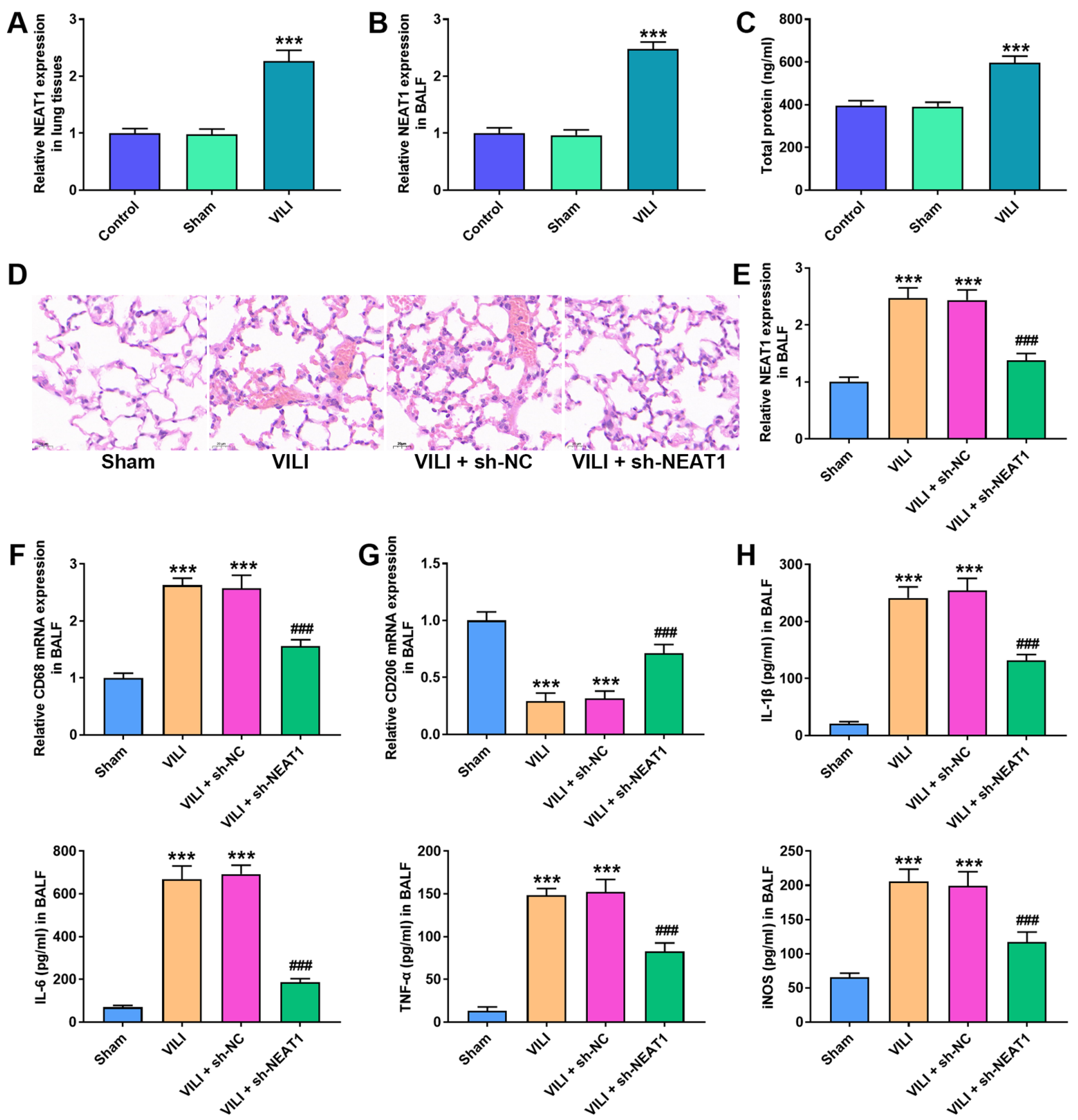

Figure 1. NEAT1 knockdown alleviates lung injury caused by VILI. RT-qPCR analysis was performed to detect NEAT1 expression in (A) lung tissues and (B) BALF. (C) The total protein level of alveolar macrophages was calculated via the BCA method. (D) The histopathological changes of lung tissues were measured via H\&E staining. (E) RT-qPCR analysis was performed to detect NEAT1 expression in BALF. RT-qPCR analysis was performed to detect the expression of the (F) M1 phenotype marker, CD86 and the (G) M2 phenotype marker, CD206 in BALF. (H) The ELISA assay was performed to detect the expression levels of IL-1 $\beta$, IL-6, TNF- $\alpha$ and iNOS. ${ }^{* * *} \mathrm{P}<0.05$ vs. the control and sham groups; ${ }^{\# \# "} \mathrm{P}<0.05$ vs. the VILI + sh-NC group. NEAT1, nuclear enriched abundant transcript 1; VILI, ventilator-induced lung injury; RT-qPCR, reverse transcription-quantitative PCR; BALF, broncho-alveolar lavage fluid; sh, short hairpin; NC, negative control; IL, interleukin, TNF, tumor necrosis factor; iNOS, inducible nitric oxide synthase.

reporter assay (Fig. 4B) showed that the luciferase activity was significantly decreased in cells co-transfected with STAT3-WT and miR-20b mimic $(\mathrm{P}<0.001)$, while no change in cells co-transfected with STAT3-MUT and miR-20b mimics were observed. The RIP assay (Fig. 4C) also demonstrated that both STAT3 and miR-20b were immunoprecipitated by anti-Ago2 $(\mathrm{P}<0.001)$. Notably, STAT3 protein expression significantly increased following transfection with pc-STAT3 compared with the pc-NC group $(\mathrm{P}<0.001$; Fig. 4D). Western blot analysis was performed to detect STAT3 protein expression in AMs following transfection with miR-20b mimics. The results demonstrated that STAT3 protein expression significantly decreased in the miR-20b mimics group compared with the mimics NC group $(\mathrm{P}<0.001$; Fig. 4E). Furthermore, western blot analysis was performed to detect STAT3 protein expression in the lung tissues of VILI mice and CS-treated AMs. As presented in Fig. 4F and G, both VILI and CS treatment significantly increased STAT3 protein expression, the effects of which were reversed following NEAT1 knockdown $(\mathrm{P}<0.001)$. 

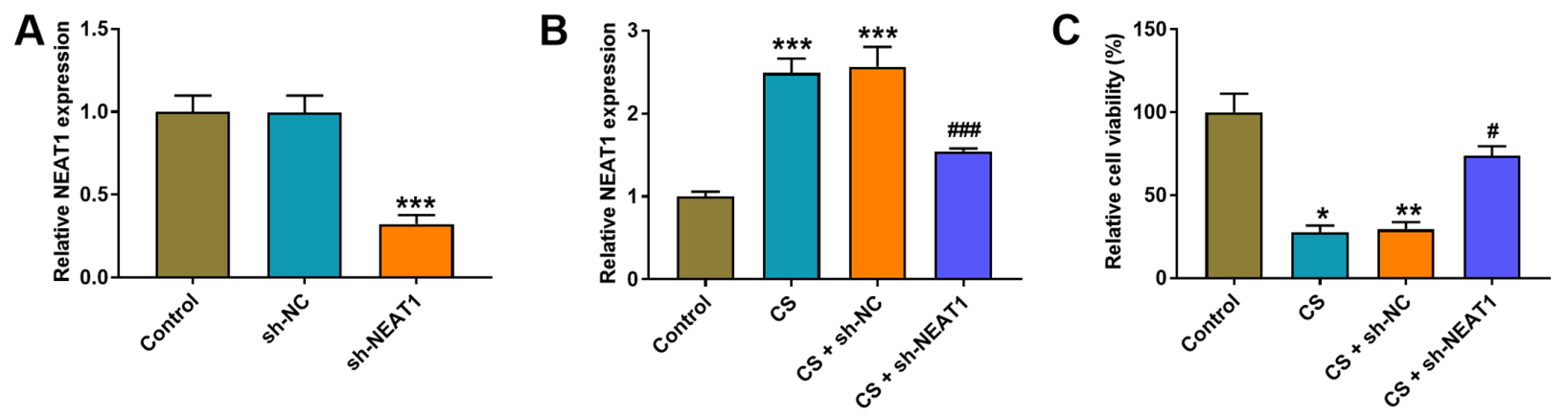

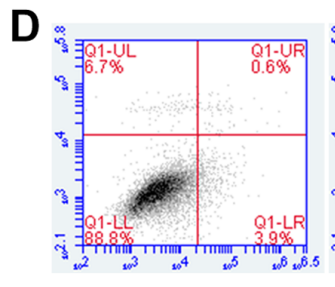

Control

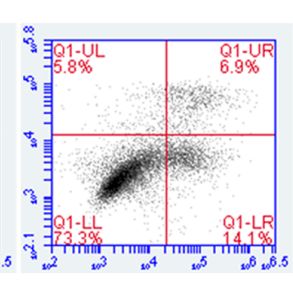

CS

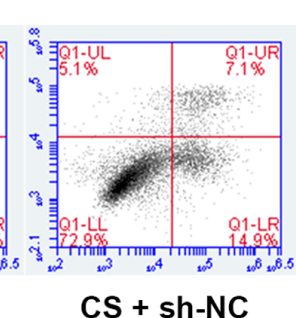

CS + sh-NC
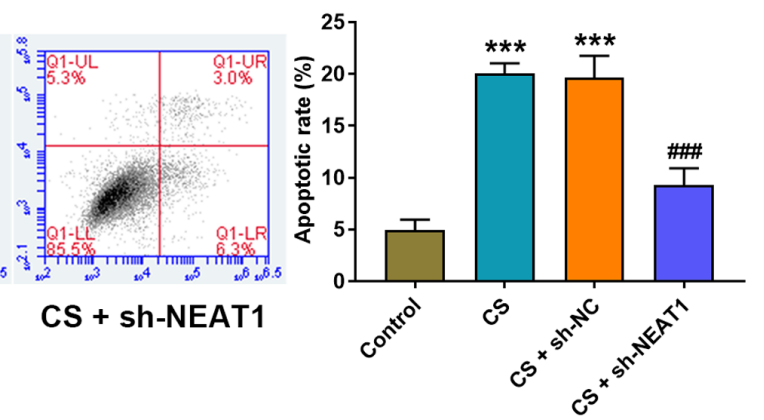

E
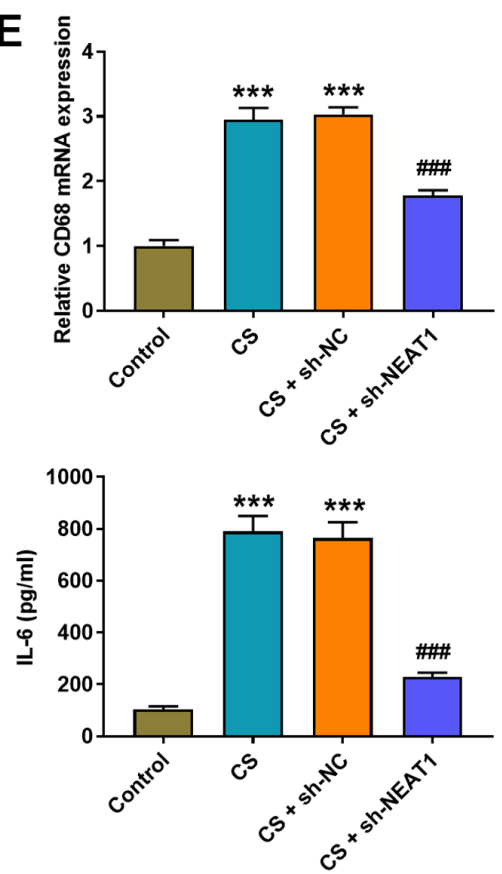

$\mathbf{F}$
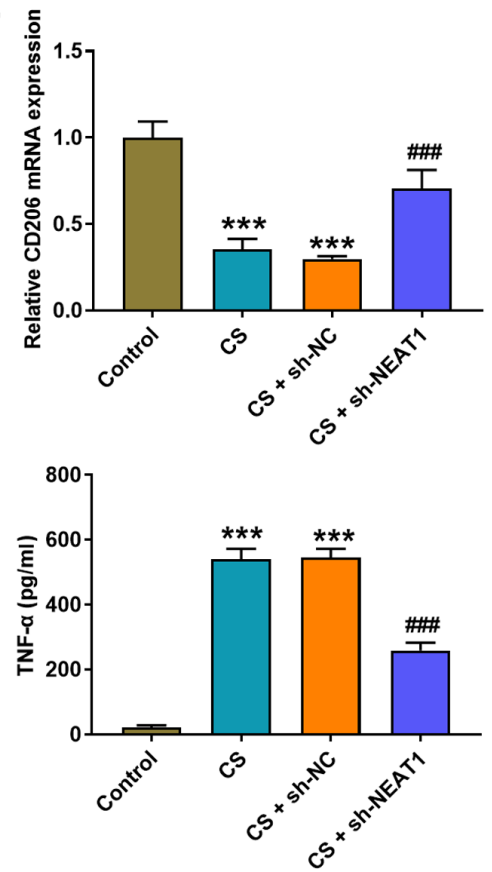

G
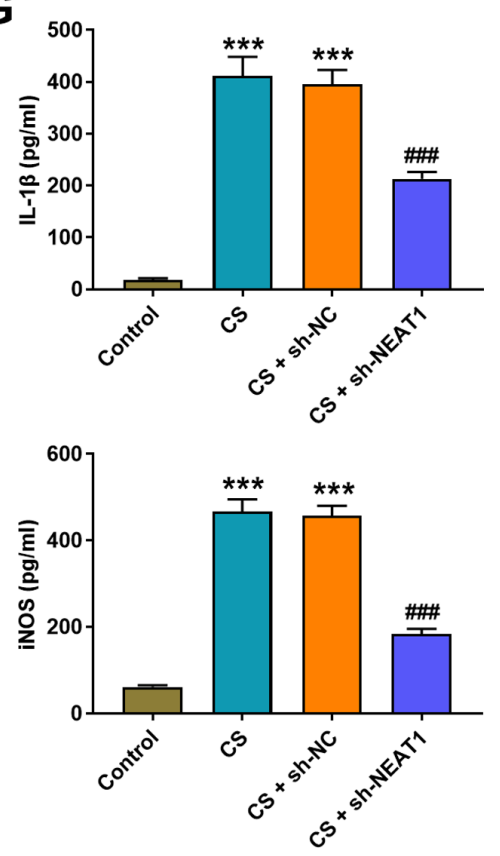

Figure 2. NEAT1 knockdown decreases the cell injury of CS treatment in AMs. (A and B) RT-qPCR analysis was performed to detect NEAT1 expression in AMs. (C) The MTT assay was performed to detect the viability of AMs. (D) Flow cytometric analysis was performed to detect the apoptosis of AMs. RT-qPCR analysis was performed to detect the expression of the (E) M1 phenotype marker, CD86 and the (F) M2 phenotype marker, CD206 in AMs. (G) The ELISA assay was performed to detect the expression levels of IL-1 $\beta$, IL-6, TNF- $\alpha$ and iNOS in AMs. ${ }^{*} \mathrm{P}<0.05,{ }^{* *} \mathrm{P}<0.01,{ }^{* * *} \mathrm{P}<0.001$ vs. the control group;

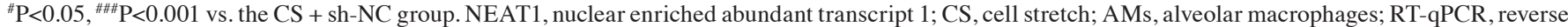
transcription-quantitative PCR; IL, interleukin, TNF, tumor necrosis factor; iNOS, inducible nitric oxide synthase; sh, short hairpin; NC, negative control.

NEAT1 knockdown alleviates CS-induced AMs injury by increasing miR-20b expression. To determine whether NEAT1 exerts its function via miR-20b, CS-treated AMs were co-transfected with NEAT1 shRNA and miR-20b inhibitor. RT-qPCR analysis demonstrated that miR-20b expression significantly increased following transfection with NEAT1 shRNA, the effects of which were reversed following transfection with miR-20b inhibitor $(\mathrm{P}<0.001$; Fig. 5A). The results of the MTT assay demonstrated that transfection with NEAT1 shRNA significantly increased the viability (Fig. 5B) and inhibited the apoptosis of AMs (Fig. 5C and D) (both $\mathrm{P}<0.001)$. Notably, these effects were reversed following transfection with miR-20b inhibitor $(\mathrm{P}<0.001$; Fig. 5B-D). RT-qPCR analysis was performed to detect the expression levels of CD68 (M1 phenotype marker) and CD206 (M2 phenotype marker) following co-transfection with NEAT1 shRNA and miR-20b inhibitor. The results demonstrated that transfection with miR-20b inhibitor increased CD68 mRNA expression (Fig. 5E) and decreased CD206 mRNA expression (Fig. 5F) compared with the sh-NEAT1 group 
A

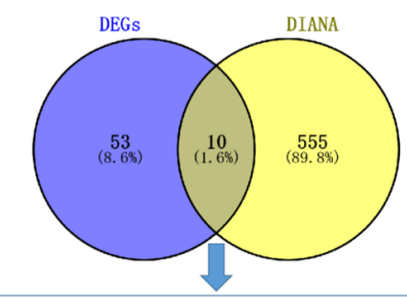

mmu-miR-7b-3p; mmu-miR-6959-5p; mmu-miR 7086-5p; mmu-miR-1955-5p; mmu-miR-5112; mmumiR-7035-3p; mmu-miR-132-3p; mmu-miR-212-3p; mmu-miR-7666-3p; mmu-miR-20b-5p
B

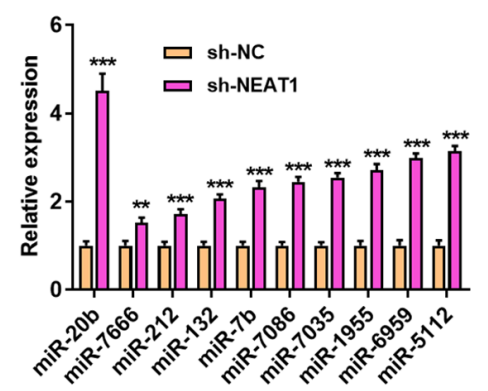

C

Target: 5' aagCUUG-GCAGCCAGCACUUUg 3'

$$
:|:||:|:|||||| \mid
$$

miRNA : 3' gaUGGACGUGAUACUCGUGAAAC 5 '

D

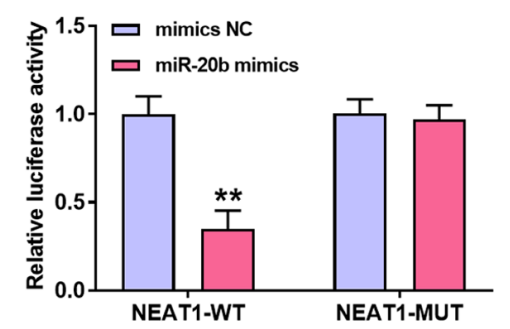

G

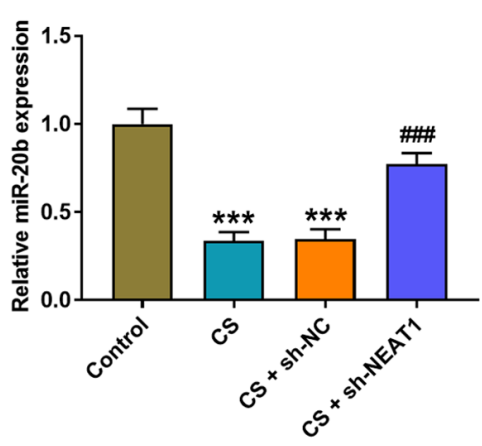

$\mathbf{E}$

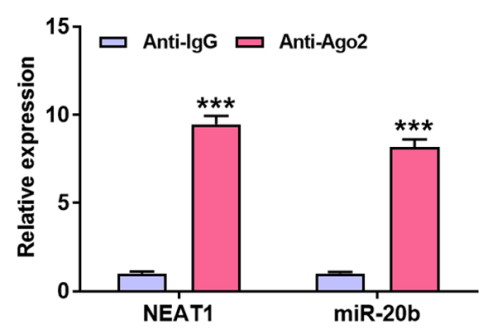

H

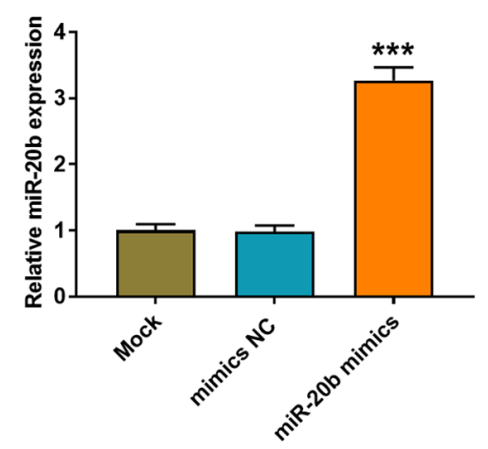

$\mathbf{F}$
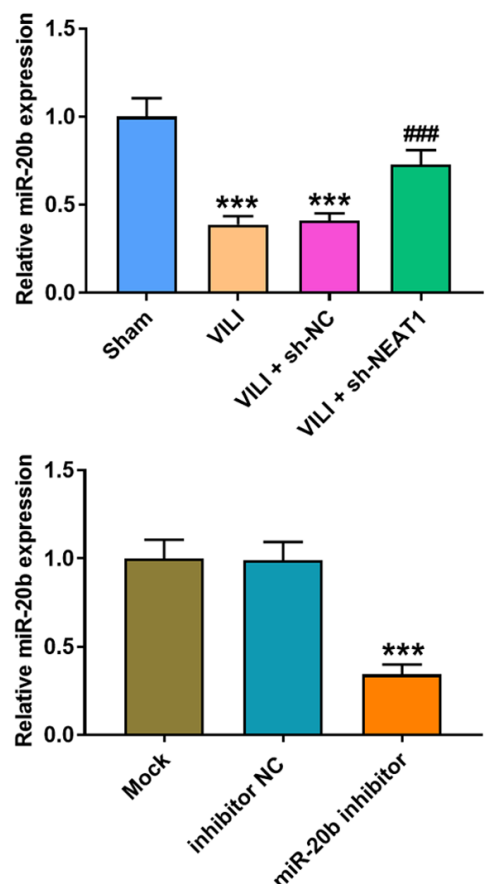

Figure 3. NEAT1 sponges miR-20b expression in AMs. (A) A total of 10 miRNAs were identified to potentially bind to NEAT1 through the intersection of DEGs and DIANA-LncBase. (B) RT-qPCR analysis was performed to detect the expression levels of the 10 miRNAs in AMs. ${ }^{* *} \mathrm{P}<0.01$, ${ }^{* * *} \mathrm{P}<0.001 \mathrm{vs}$. the sh-NC group. (C) The binding site between NEAT1 and miR-20b was predicted using the StarBase 3.0 database. (D) Luciferase activity was determined via the dual-luciferase reporter gene assay. ${ }^{* *} \mathrm{P}<0.01$ vs. the mimics NC group. (E) The RIP assay was performed to assess the interaction between NEAT1 and miR-20b. ${ }^{* * *} \mathrm{P}<0.001$ vs. the anti-lgG group. RT-qPCR analysis was performed to detect miR-20b expression in the lung tissues of (F) VILI mice and (G) CS treated AMs. ${ }^{* * *} \mathrm{P}<0.001$ vs. the sham and control groups; ${ }^{\# \# ~} \mathrm{P}<0.001$ vs. the VILI + sh-NC group and CS + sh-NC groups. (H) RT-qPCR analysis was performed to detect miR-20b expression in AMs transfected with miR-20b mimics or miR-20b inhibitor. ${ }^{* * *} \mathrm{P}<0.001$ vs. the mimics NC and the inhibitor NC groups. NEAT1, nuclear enriched abundant transcript 1; miR/miRNA, microRNA; AMs, alveolar macrophages; DEGs. differentially expressed genes; RT-qPCR, reverse transcription-quantitative PCR; VILI, ventilator-induced lung injury; CS, cell stretch; sh, short hairpin; NC, negative control; WT, wild-type; MUT, mutant.

(both $\mathrm{P}<0.001$ ), which suggests that low miR-20b expression enhances polarization into M1 and inhibits polarization into M2. The results of the ELISA assay demonstrated that transfection with miR-20b inhibitor increased the expression levels of IL-1 $\beta$, IL-6, TNF- $\alpha$ and iNOS, the effects of which were reversed following NEAT1 knockdown $(\mathrm{P}<0.001$; Fig. 5G).

Overexpression of STAT3 eliminates the protective effect of miR-20b on cell injury of CS-induced AMs. To verify whether miR-20b exerts its function via STAT3, CS-treated AMs were co-transfected with miR-20b mimics and STAT3 overexpressed plasmid (pcDNA3.1 STAT3). Western blot analysis demonstrated that STAT3 protein expression significantly decreased following transfection with miR-20b mimics, the effects of which were reversed following overexpression of STAT3 $(\mathrm{P}<0.001$; Fig. 6A). In addition, transfection with miR-20b mimics significantly increased the viability (Fig. 6B) and inhibited the apoptosis (Fig. 6C) of AMs (both $\mathrm{P}<0.001$ ). Conversely, overexpression of STAT3 significantly inhibited the viability (Fig. 6B) and induced the apoptosis (Fig. 6C) of AMs (both $\mathrm{P}<0.001)$. Notably, transfection with miR-20b mimics significantly decreased CD68 mRNA expression (Fig. 6D) and increased CD206 mRNA expression (Fig. 6E) in CS-treated AMs, the effects of which were reversed following overexpression of STAT3 $(\mathrm{P}<0.001)$. The results of the ELISA assay demonstrated that transfection with miR-20b mimics 


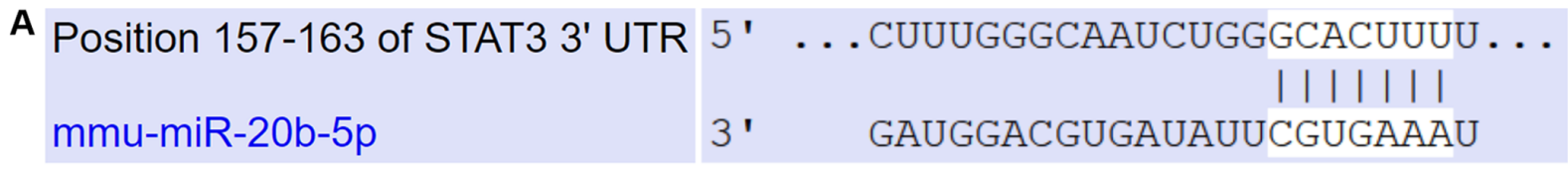

B

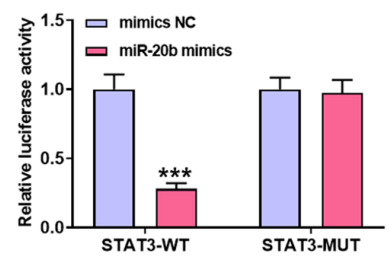

E
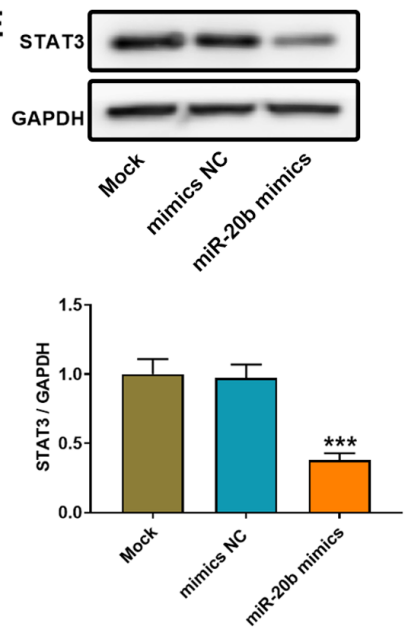

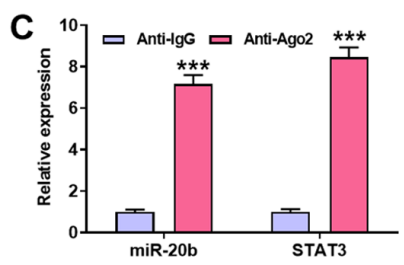

$\mathbf{F}$
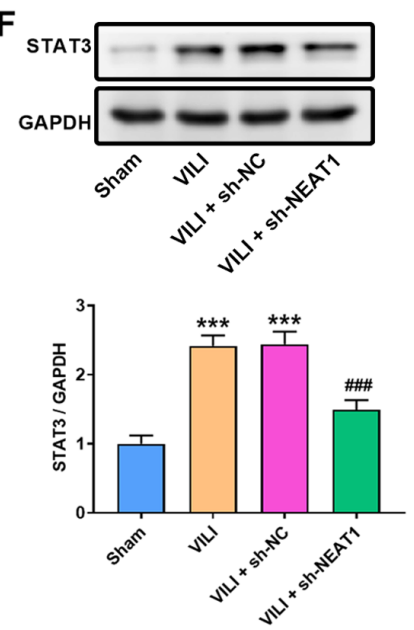
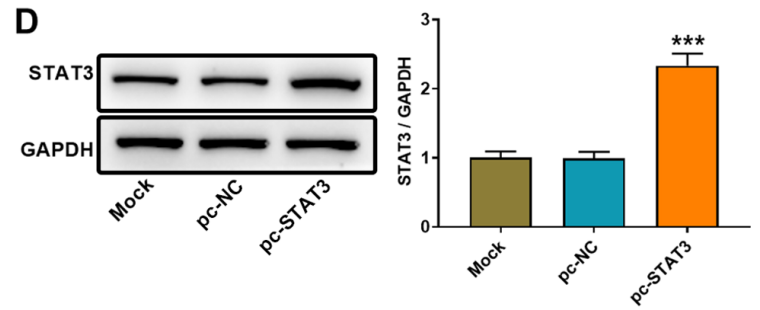

G
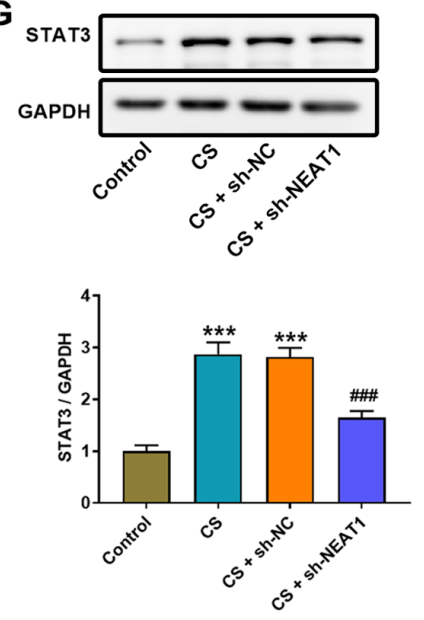

Figure 4. NEAT1 modulates miR-20b expression to regulate STAT3 expression in AMs. (A) The binding site between miR-20b and STAT3 was predicted using TargetScan. (B) Luciferase activities were determined via the dual-luciferase reporter assay. ${ }^{* * *} \mathrm{P}<0.001 \mathrm{vs}$. the mimics NC group. (C) The RIP assay was performed to assess the interaction between NEAT1 and miR-20b. ${ }^{* * *} \mathrm{P}<0.001$ vs. the anti-lgG group. (D) Western blot analysis was performed to detect STAT3 protein expression in AMs. ${ }^{* * *} \mathrm{P}<0.001$ vs. the pc-NC group. (E) Western blot analysis was performed to detect STAT3 protein expression following transfection with miR-20b mimics or NC mimics. ${ }^{* * *} \mathrm{P}<0.001$ vs. the mimics NC group. Western blot analysis was performed to detect STAT3 protein expression in the lung tissues of (F) VILI mice or (G) CS-treated AMs. ${ }^{* * *} \mathrm{P}<0.001$ vs. the sham and control groups; ${ }^{\# \#} \mathrm{P}<0.001 \mathrm{vs}$. the VILI + sh-NC and CS + sh-NC groups. NEAT1, nuclear enriched abundant transcript 1; miR, microRNA; AMs, alveolar macrophages; NC, negative control; VILI, ventilator-induced lung injury; CS, cell stretch; sh, short hairpin; UTR, untranslated region; WT, wild-type; MUT, mutant.

decreased the expression levels of IL-1 $\beta$, IL- 6 , TNF- $\alpha$ and iNOS in AMs, the effects of which were reversed following overexpression of STAT3 (all P<0.001; Fig. 6F). The aforementioned results indicated that lncRNA NEAT1 regulated the expression of STAT3 by targeting miR-20b, thereby affecting the polarization of alveolar macrophages in VILI mice and increasing inflammation and lung injury (Fig. 7).

\section{Discussion}

MV is an essential therapeutic tool for patients with acute respiratory distress syndrome $(1,29)$. However, MV can also induce or exacerbate lung injury (29). The present study aimed to investigate the protective effect of NEAT1 knockdown in the in vitro and in vivo model of VILI. The results demonstrated that NEAT1 knockdown promoted the phenotypic transformation of AMs from the pro-inflammatory (M1) to the anti-inflammatory (M2) phenotype, and reduced inflammation by regulating miR-20b and STAT3 expression.

Previous studies have demonstrated that regulating the expression of lncRNAs can alleviate lung injury (30-32). Wang et al (33) reported that CASC9 is significantly downregulated in human small airway epithelial cells (HSAECs) treated with LPS, and in the lung tissues of rats with sepsis, whereas overexpression of CASC9 significantly promotes the viability of HSAECs. MALAT1 is highly expressed in lung transplant ischemia-reperfusion (LTIR), and MALAT1 knockdown ameliorates injury following LTIR (34). The results of the present study demonstrated that NEAT1 expression was significantly upregulated in the lung tissues of VILI mice and CS-treated AMs. NEAT1 knockdown weakened the histopathological injury of the lung tissues, decreased apoptosis of AMs and decreased the number of macrophages. Furthermore, NEAT1 knockdown alleviated lung injury induced by VILI and suppressed apoptosis in CS-treated AMs.

The interaction between miRNAs and IncRNAs is indispensable in the development of diseases $(32,35,36)$. Tang et al (26) reported that as the ceRNA for miR-20b-5p, HOTAIR knockdown attenuates autophagy in hepatic I/R injury by improving the inhibitory effect of miR-20b-5p on ATG7. Furthermore, You et al (37) demonstrated that miR-20b expression was downregulated in a CCI rat model, and miR-20b-5p mimics alleviates neuropathic pain by inhibiting Akt3 expression in CCI rats, which supports the results 

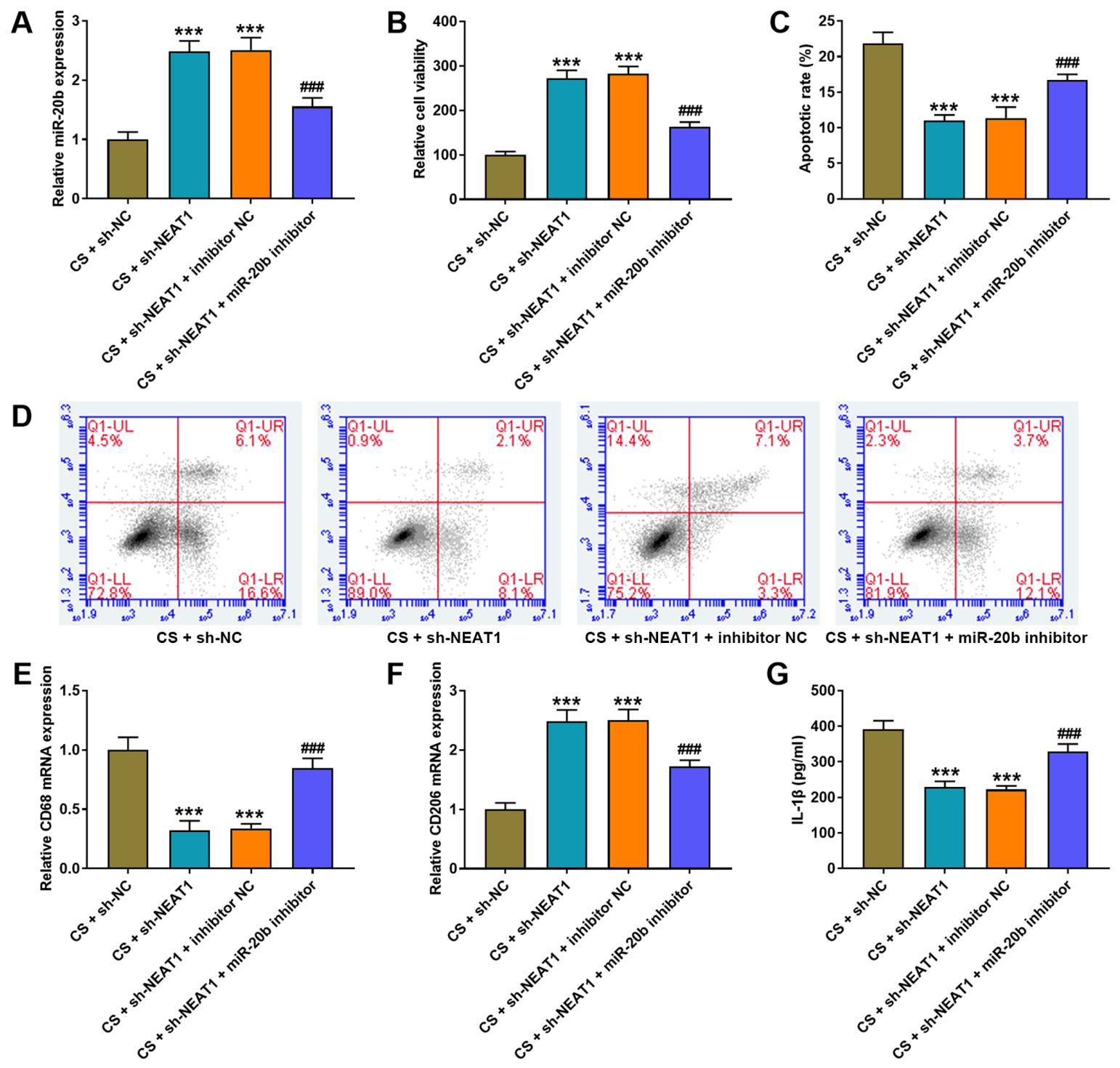

F
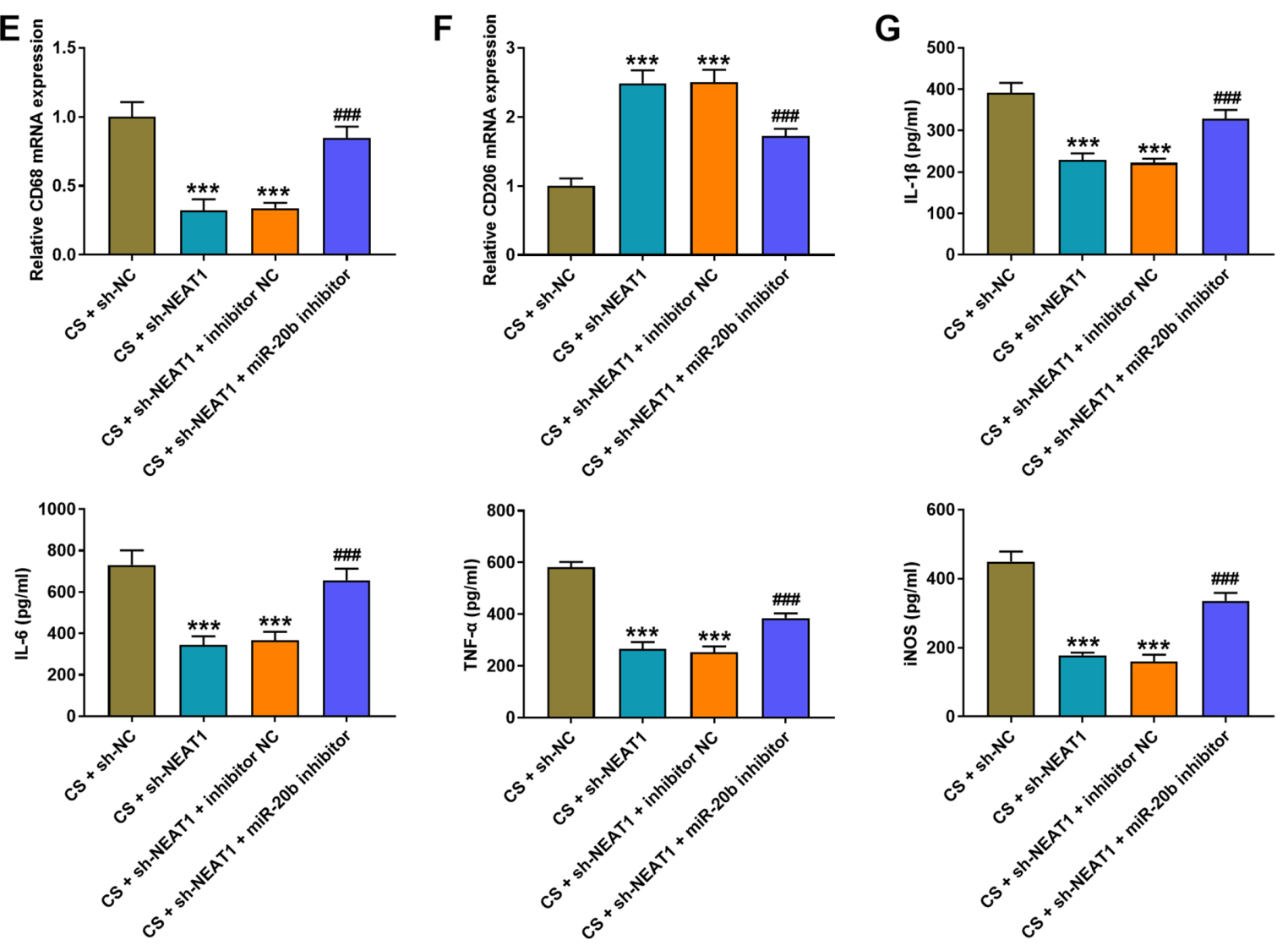

Figure 5. NEAT1 knockdown alleviates CS-induced AMs injury by increasing miR-20b expression. (A) RT-qPCR analysis was performed to detect miR-20b expression in AMs following co-transfection with NEAT1 shRNA and miR-20b inhibitor. (B) The MTT assay was performed to assess the viability of AMs following co-transfection with NEAT1 shRNA and miR-20b inhibitor. (C and D) Flow cytometric analysis was performed to detect the apoptosis of AMs following co-transfection with NEAT1 shRNA and miR-20b inhibitor. RT-qPCR analysis was performed to detect the expression of (E) the M1 phenotype marker, CD86 and (F) the M2 phenotype marker, CD206 in AMs following co-transfection with NEAT1 shRNA and miR-20b inhibitor. (G) The ELISA assay was performed to detect the expression levels of IL-1 $\beta$, IL-6, TNF- $\alpha$ and iNOS in AM following co-transfection with NEAT1 shRNA and miR-20b inhibitor. ${ }^{* * * *} \mathrm{P}<0.001$ vs. the CS + sh-NC group; ${ }^{\# \# \# ~} \mathrm{P}<0.001$ vs. the CS + sh-NEAT1 + inhibitor NC group. NEAT1, nuclear enriched abundant transcript 1; CS, cell stretch; AMs, alveolar macrophages; miR, microRNA; RT-qPCR, reverse transcription-quantitative PCR; sh, short hairpin; NC, negative control; IL, interleukin; TNF, tumor necrosis factor; iNOS, inducible nitric oxide synthase.

of the present study. In the present study, miR-20b expression was downregulated in both the lung tissues of VILI mice and
CS-treated AMs. The dual-luciferase reporter and RIP assays confirmed that miR-20b is the target miRNA of NEAT1. 
A

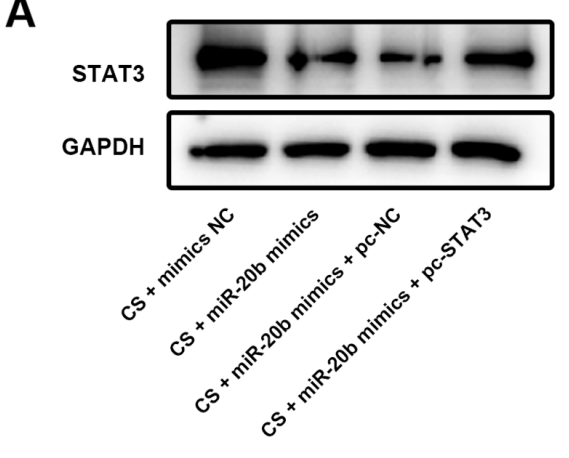

C

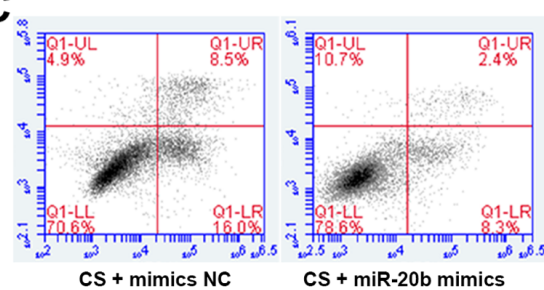

D
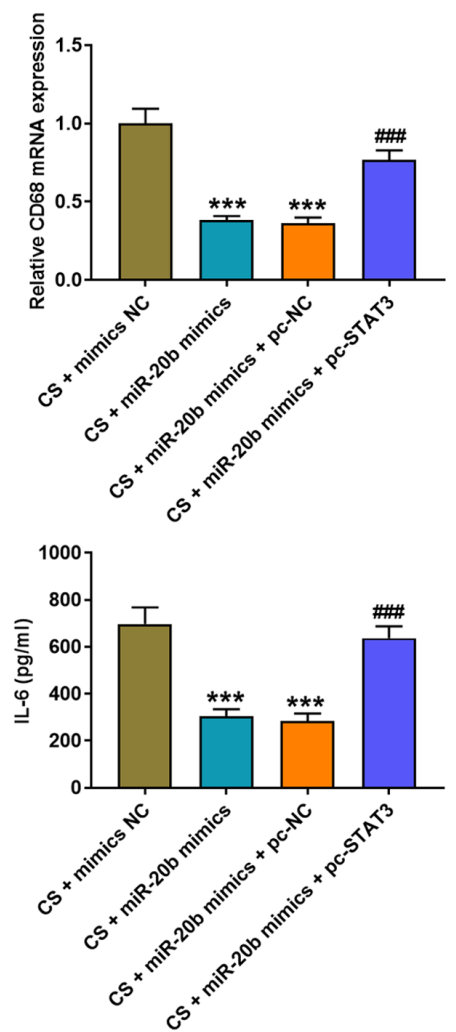

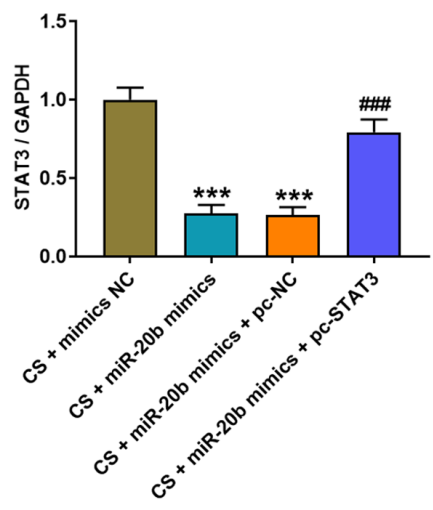

B
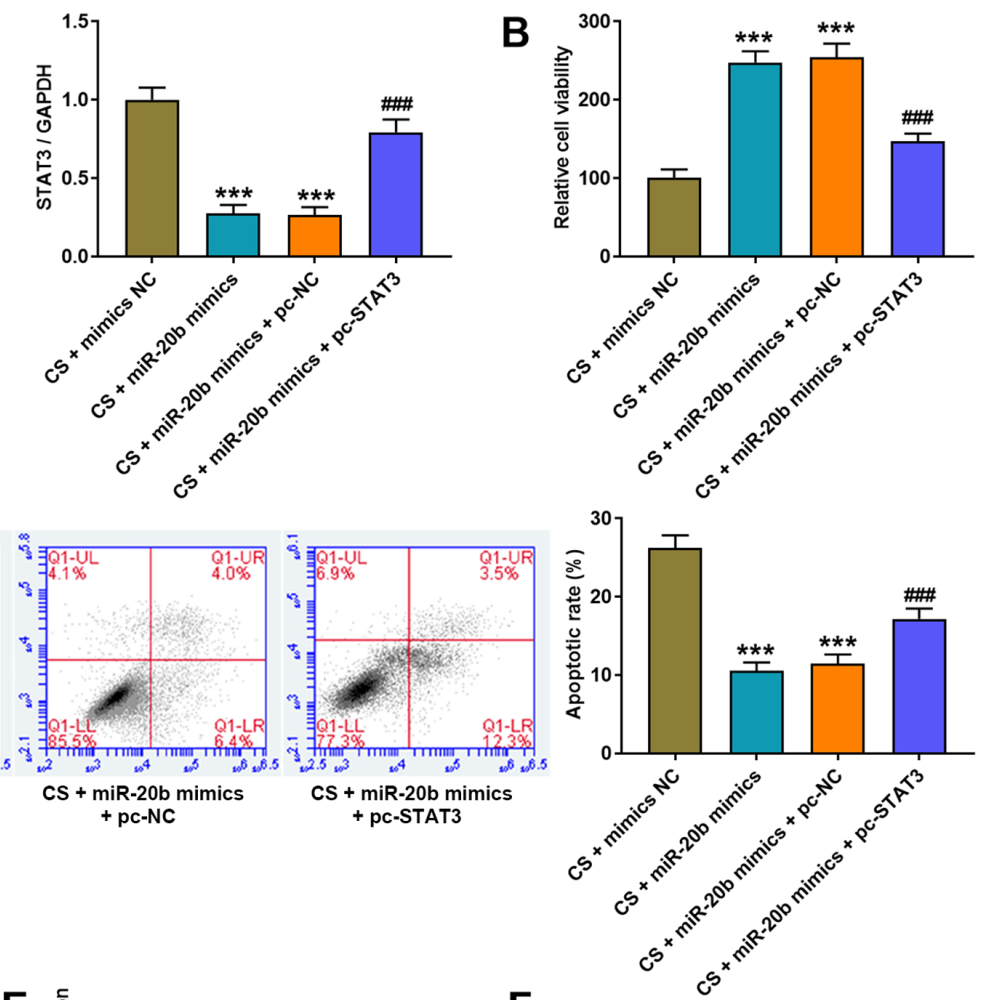

F
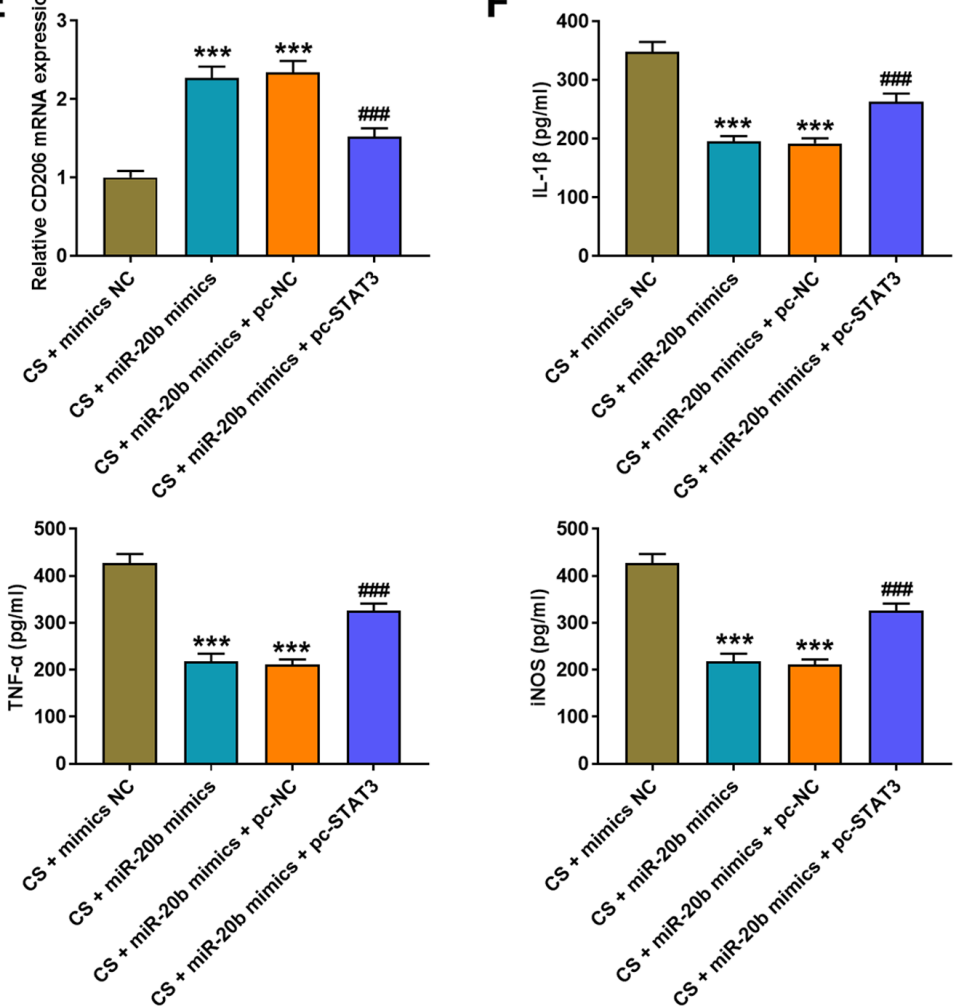

E
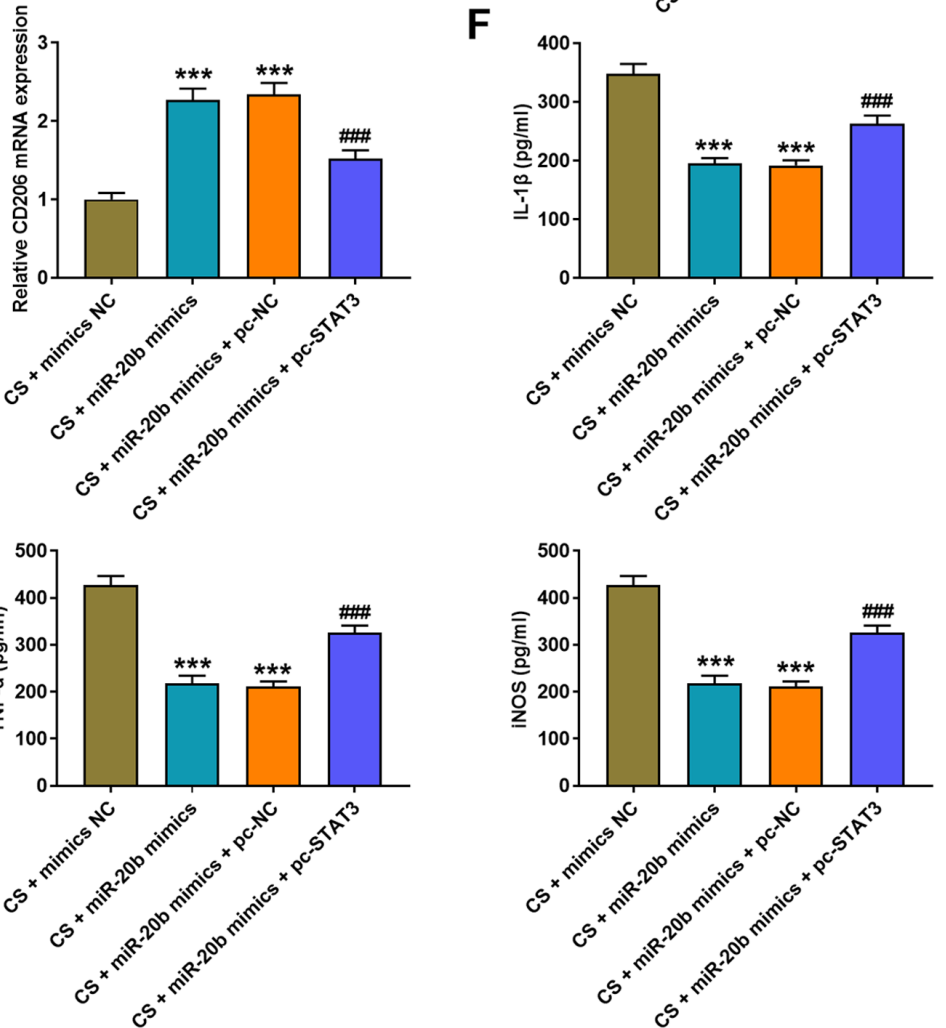

Figure 6. Overexpression of STAT3 eliminates the protected effect of miR-20b on cell injury of CS-induced AMs. (A) Western blot analysis was performed to detect STAT3 protein expression in AMs following co-transfection with miR-20b mimics and pcDNA3.1 STAT3. (B) The MTT assay was performed to detect the viability of AMs following co-transfection with miR-20b mimics and pcDNA3.1 STAT3. (C) Flow cytometric analysis was performed to assess the apoptosis of AMs following co-transfection with miR-20b mimics and pcDNA3.1 STAT3. Reverse transcription-quantitative PCR analysis was performed to detect the expression of (D) the M1 phenotype marker, CD86 and (E) the M2 phenotype marker, CD206 in AMs following co-transfection with miR-20b mimics and pcDNA3.1 STAT3. (F) The ELISA assay was performed to detect the expression levels of IL-1 $\beta$, IL-6, TNF- $\alpha$ and iNOS in AMs following co-transfection with miR-20b mimics and pcDNA3.1 STAT3. ${ }^{* * * *} \mathrm{P}<0.001$ vs. the CS + mimics NC group; ${ }^{\# \# ~} \mathrm{P}<0.001$ vs. the CS + miR-20b mimics + pc-NC group. miR, microRNA; CS, cell stretch; AMs, alveolar macrophages; IL, interleukin; TNF, tumor necrosis factor; iNOS, inducible nitric oxide synthase; NC, negative control.

Notably, the inhibitory effect of sh-NEAT1 on apoptosis, CD68 expression and the expression levels of IL-1 $\beta$, IL-6,
TNF- $\alpha$ and iNOS was eliminated following transfection with miR-20b inhibitor. Taken together, these results suggest that 


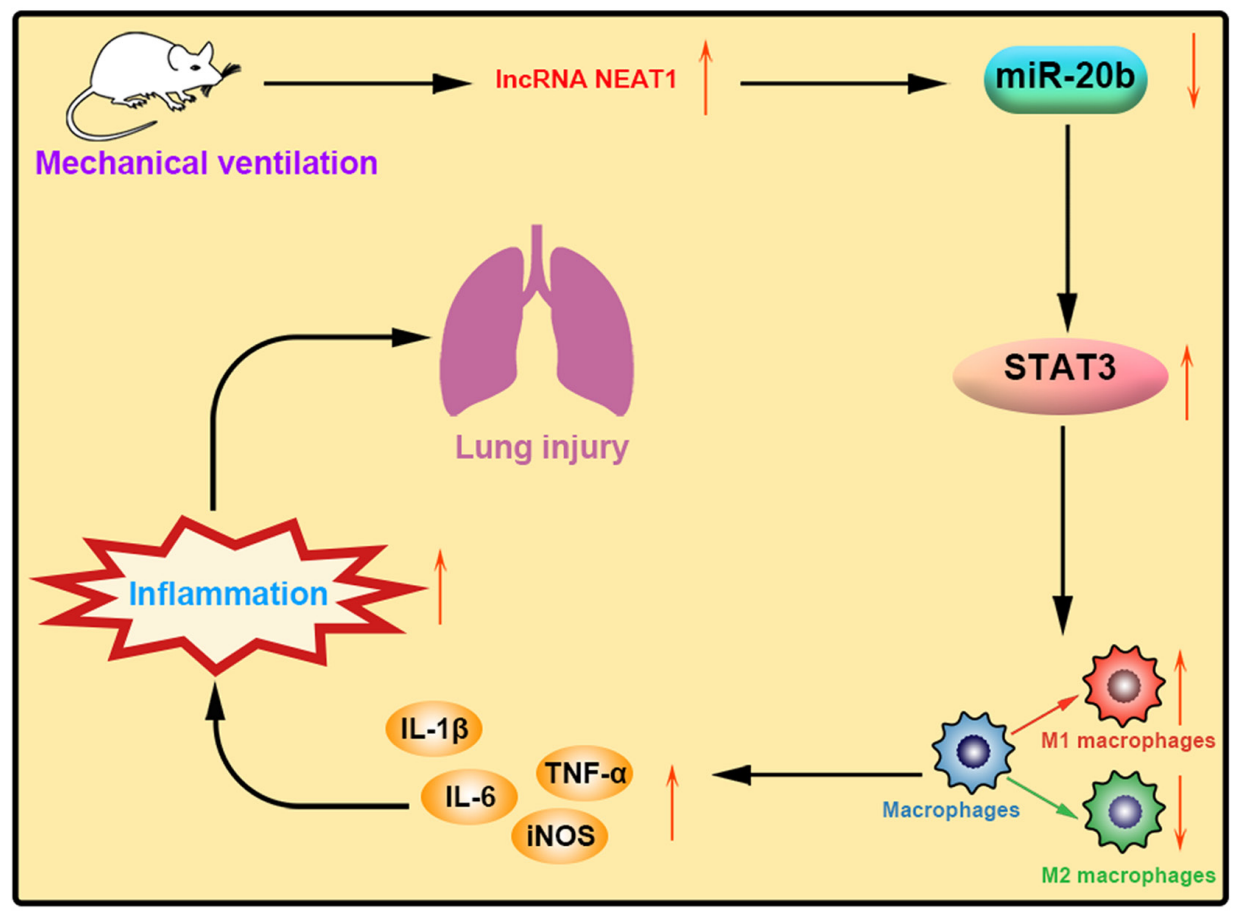

Figure 7. Schematic model of lncRNA NEAT1 function in VILI. NEAT1 regulates STAT3 expression by targeting miR-20b, which affects the polarization of alveolar macrophages, increasing inflammation and lung injury in VILI mice. IncRNA, long non-coding RNA; NEAT1, nuclear enriched abundant transcript 1; VILI, ventilator-induced lung injury; miR, microRNA; IL, interleukin; TNF, tumor necrosis factor; iNOS, inducible nitric oxide synthase.

NEAT1 may inhibit the progression of VILI by upregulating miR-20b expression.

Previous studies have reported that STAT3 is implicated in the pathogenesis of alcoholic hepatitis (AH) and lung injury $(38,39)$. Facilitation of STAT3 phosphorylation in AMs exacerbates pulmonary inflammation in acute lung injury (ALI) and aggravates lung injury in ALI rats (39). STAT3 is activated in mice with $\mathrm{AH}$, inhibition of which alleviates liver injury (38). The results of the present study demonstrated that STAT3 was highly expressed in the lung tissues of VILI mice and CS-treated AMs. The results confirmed that STAT3 is the direct target of miR-20b, and was positively regulated by NEAT1. STAT3 expression decreased following transfection with both miR-20b mimics and sh-NEAT1, the effects of which were reversed following transfection with miR-20b inhibitor. Collectively, these results suggest that NEAT1 knockdown may inhibit the progression of VILI by regulating miR-20b-mediated STAT3.

Recent studies have reported that activated macrophages play a vital role in the progression of several inflammatory diseases (30,40-42). The M1 and M2 phenotypes play opposing roles in the regulation of inflammation. M1 macrophages play important roles in immune inflammatory effects, whereas M2 macrophages mainly exert anti-inflammatory effects $(43,44)$. In acute spinal cord injury (SCI) mice, lncGBP9 knockdown promotes M2 polarization of the macrophages, which reduces the inflammatory levels and facilitates repair following SCI (41). Ji et al (45) demonstrated that promoting the transformation of M2 macrophages can protect the podocytes from high-glucose-induced injury in DN, which supports the results of the present study. CD68 mRNA expression (M1 phenotype marker) increased and CD206 mRNA expression (M2 phenotype marker) decreased in the lung tissues of VILI mice, and NEAT1 knockdown inhibited CD68 expression and enhanced CD206 expression. Similar results were observed in CS-treated AMs. Notably, the expression levels of IL-1 $\beta$, IL-6, TNF- $\alpha$ and iNOS were decreased in VILI mice and CS-treated AMs concomitant with the increasing transformation of M2 macrophages. Taken together, these results suggest that NEAT1 knockdown may remit injury of VILI by promoting the phenotypic transformation of AMs from M1 to M2.

In conclusion, the results of the present study demonstrated that NEAT1 expression was upregulated in the lung tissues of VILI mice and CS-treated AMs, and played an important role in accelerating the progress of VILI. NEAT1 knockdown promoted the phenotypic transformation of AMs from M1 to M2, thereby suppressing inflammatory levels and reducing histopathological injury and apoptosis of VILI. The regulatory effect of NEAT1 on VILI may be achieved by regulating miR-20b and STAT3 expression. However, the present study did not explore whether there are other targets that interact with NEAT1 in VILI, and investigation into its underlying mechanisms require further research in the future.

\section{Acknowledgements}

Not applicable.

\section{Funding}

No funding was received.

\section{Availability of data and materials}

The datasets used and/or analyzed during the current study are available from the corresponding author on reasonable request. 


\section{Authors' contributions}

YL designed the present study and performed the literature review. YL and GT performed the experiments. GT and JL analyzed the data and drafted the initial manuscript. GT and JL confirm the authenticity of all the raw data. All authors have read and approved the final manuscript.

\section{Ethics approval and consent to participate}

All animal experiments were approved by the Affiliated Huaian No.1 People's Hospital of Nanjing Medical University (Huaian, China; approval no. HASDY20201009) and performed in accordance with the Guide for the Care and Use of Laboratory Animals.

\section{Patient consent for publication}

Not applicable.

\section{Competing interests}

The authors declare that they have no competing interests.

\section{References}

1. Gattinoni L, Tonetti T, Cressoni M, Cadringher P, Herrmann P, Moerer O, Protti A, Gotti M, Chiurazzi C, Carlesso E, et al: Ventilator-related causes of lung injury: The mechanical power. Intensive Care Med 42: 1567-1575, 2016.

2. Fan E, Brodie D and Slutsky AS: Acute Respiratory Distress Syndrome: Advances in Diagnosis and Treatment. JAMA 319: 698-710, 2018

3. Xu B, Wang Y, Li X, Mao Y and Deng X: RNA sequencing analysis of aberrantly expressed long non coding RNAs and mRNAs in a mouse model of ventilator induced lung injury. Mo Med Rep 18: 882-892, 2018.

4. Zhang B, Zhang X, Li Q, Ma F, Sun L and Wang M: Dexmedetomidine attenuates ventilator-induced lung injury in rats by up-regulating NLRC3. Ann Palliat Med 9: 2474-2484, 2020.

5. SunZ, Wang F, Yang Y, Wang J, Sun S, Xia H and Yao S: Resolvin D1 attenuates ventilator-induced lung injury by reducing HMGB1 release in a HO-1-dependent pathway. Int Immunopharmacol 75 $105825,2019$.

6. Veskemaa L, Graw JA, Pickerodt PA, Taher M, Boemke W, González-López A and Francis RC: Tert-butylhydroquinone augments Nrf2-dependent resilience against oxidative stress and improves survival of ventilator-induced lung injury in mice. Am J Physiol Lung Cell Mol Physiol 320: L17-L28, 2021.

7. Lin JY, Jing R, Lin F, Ge WY, Dai HJ and Pan L: High tidal volume induces mitochondria damage and releases mitochondrial dna to aggravate the ventilator-induced lung injury. Front Immunol 9: 1477, 2018.

8. Dai H, Zhang S, Du X, Zhang W, Jing R, Wang X and Pan L: RhoA inhibitor suppresses the production of microvesicles and rescues high ventilation induced lung injury. Int Immunopharmacol 72 : 74-81, 2019.

9. Matthay MA and Zimmerman GA: Acute lung injury and the acute respiratory distress syndrome: Four decades of inquiry into pathogenesis and rational management. Am J Respir Cell Mol Biol 33: 319-327, 2005.

10. Gautier EL, Shay T, Miller J, Greter M, Jakubzick C, Ivanov S Helft J, Chow A, Elpek KG, Gordonov S, et al; Immunological Genome Consortium: Gene-expression profiles and transcriptional regulatory pathways that underlie the identity and diversity of mouse tissue macrophages. Nat Immunol 13: 1118-1128, 2012

11. Wynn TA, Chawla A and Pollard JW: Macrophage biology in development, homeostasis and disease. Nature 496: 445-455, 2013

12. Mosser D and Edwards J: Exploring the full spectrum of macrophage activation. Nat Rev Immunol 8:958-969. Nat Rev Immunol 8: 958-969, 2008
13. Safavian D, Leung CH, Kapus A, Ailenberg M, Szaszi K, Shani R, Di Ciano-Oliveira C, Ghazarian M and Rotstein O: Hemorrhagic shock/resuscitation reduces the M2 phenotype of alveolar macrophages: A potential mechanism contributing to increased LPS-induced lung injury. Shock 51: 213-220, 2019.

14. Genin M, Clement F, Fattaccioli A, Raes M and Michiels C: M1 and M2 macrophages derived from THP-1 cells differentially modulate the response of cancer cells to etoposide. BMC Cancer 15: 577, 2015.

15. Brown BN, Ratner BD, Goodman SB, Amar S and Badylak SF: Macrophage polarization: An opportunity for improved outcomes in biomaterials and regenerative medicine. Biomaterials 33: 3792-3802, 2012

16. Biswas SK and Mantovani A: Macrophage plasticity and interaction with lymphocyte subsets: Cancer as a paradigm. Nat Immunol 11: 889-896, 2010.

17. Chistiakov DA, Bobryshev YV, Nikiforov NG, Elizova NV, Sobenin IA and Orekhov AN: Macrophage phenotypic plasticity in atherosclerosis: The associated features and the peculiarities of the expression of inflammatory genes. Int J Cardiol 184: 436-445, 2015.

18. Yao Z, Jia X, Megger DA, Chen J, Liu Y, Li J, Sitek B and Yuan Z: Label-free proteomic analysis of exosomes secreted from THP-1-derived macrophages treated with IFN- $\alpha$ identifies antiviral proteins enriched in exosomes. J Proteome Res 18: 855-864, 2019.

19. Qiao Y, Peng C, Li J, Wu D and Wang X: LncRNA MALAT1 is neuroprotective in a rat model of spinal cord Ischemia-Reperfusion injury through miR-204 regulation. Curr Neurovasc Res 15: 211-219, 2018.

20. Fang Y, Hu J, Wang Z, Zong H, Zhang R and Sun L: LncRNA H19 functions as an Aquaporin 1 competitive endogenous RNA to regulate microRNA-874 expression in LPS sepsis. Biomed Pharmacother 105: 1183-1191, 2018.

21. Yamamura S, Imai-Sumida $\mathbf{M}$, Tanaka $\mathrm{Y}$ and Dahiya $\mathrm{R}$ : Interaction and cross-talk between non-coding RNAs. Cell Mol Life Sci 75: 467-484, 2018

22. Guo D, Ma J, Yan L,Li T, Li Z, Han X and Shui S: Down-regulation of Lncrna MALAT1 attenuates neuronal cell death through suppressing Beclin1-dependent autophagy by regulating Mir-30a in cerebral ischemic stroke. Cell Physiol Biochem 43: 182-194, 2017.

23. Wang W, Jia YJ, Yang YL, Xue M, Zheng ZJ, Wang L and Xue YM: LncRNA GAS5 exacerbates renal tubular epithelial fibrosis by acting as a competing endogenous RNA of miR-96-5p. Biomed Pharmacother 121: 109411, 2020.

24. Wang X, Xu Y, Zhu YC, Wang YK, Li J, Li XY, Ji T and Bai SJ: LncRNA NEAT1 promotes extracellular matrix accumulation and epithelial-to-mesenchymal transition by targeting miR-27b-3p and ZEB1 in diabetic nephropathy. J Cell Physiol 234: 12926-12933, 2019.

25. Zhou H, Wang X and Zhang B: Depression of lncRNA NEAT1 antagonizes LPS-evoked acute injury and inflammatory response in alveolar epithelial cells via HMGB1-RAGE signaling. Mediators Inflamm 2020: 8019467, 2020.

26. Tang B, Bao N, He G and Wang J: Long noncoding RNA HOTAIR regulates autophagy via the miR-20b-5p/ATG7 ax is in hepatic ischemia/reperfusion injury. Gene 686: 56-62, 2019.

27. Wang Y, Minshall RD, Schwartz DE and Hu G: Cyclic stretch induces alveolar epithelial barrier dysfunction via calpain-mediated degradation of p120-catenin. Am J Physiol Lung Cell Mol Physiol 301: L197-L206, 2011.

28. Livak KJ and Schmittgen TD: Analysis of relative gene expression data using real-time quantitative PCR and the 2(-Delta Delta C(T)) method. Methods 25: 402-408, 2001.

29. Ding H, Wang Y, Dong W, Ren R, Mao Y and Deng X: Proteomic lung analysis of mice with ventilator-induced lung injury (VILI) using iTRAQ-based quantitative proteomics. Chem Pharm Bull (Tokyo) 66: 691-700, 2018.

30. Cui H, Banerjee S, Guo S, Xie N, Ge J, Jiang D, Zörnig M, Thannickal VJ and Liu G: Long noncoding RNA Malat1 regulates differential activation of macrophages and response to lung injury. JCI Insight 4: e124522, 2019.

31. Song X, Li L, Zhao Y and Song Y: Down-regulation of long non-coding RNA XIST aggravates sepsis-induced lung injury by regulating miR-16-5p. Hum Cell 34: 1335-1345, 2021.

32. Li X, Mo J, Li J and Chen Y: IncRNA CASC2 inhibits lipopolysaccharide induced acute lung injury via miR 27b/TAB2 axis. Mol Med Rep 22: 5181-5190, 2020.

33. Wang HR, Guo XY, Liu XY and Song X: Down-regulation of lncRNA CASC 9 aggravates sepsis-induced acute lung injury by regulating miR-195-5p/PDK4 axis. Inflamm Res 69: 559-568, 2020. 
34. Wei L, Li J, Han Z, Chen Z and Zhang Q: Silencing of lncRNA MALAT1 prevents inflammatory injury after lung transplant ischemia-reperfusion by downregulation of IL-8 via p300. Mol Ther Nucleic Acids 18: 285-297, 2019.

35. Liu M, Li W, Song F, Zhang L and Sun X: Silencing of lncRNA MIAT alleviates LPS-induced pneumonia via regulating miR-147a/ NKAP/NF- $\kappa$ B axis. Aging (Albany NY) 13: 2506-2518, 2020.

36. Zhang M, Cheng K, Chen H, Tu J, Shen Y, Pang L, Wu W and Yu Z: LncRNA AK020546 protects against cardiac ischemiareperfusion injury by sponging miR-350-3p. Aging (Albany NY) 13: 14219-14233, 2021.

37. You H, Zhang L, Chen Z, Liu W, Wang H and He H: MiR-20b-5p relieves neuropathic pain by targeting Akt3 in a chronic constriction injury rat model. Synapse 73: e22125, 2019.

38. Wan Y-M, Li Z-Q, Zhou Q, Liu C, Wang MJ, Wu HX, Mu YZ, He YF, Zhang Y, Wu XN, et al: Mesenchymal stem cells alleviate liver injury induced by chronic-binge ethanol feeding in mice via release of TSG6 and suppression of STAT3 activation. Stem Cell Res Ther 11: 24-24, 2020

39. Wu J, Yan X and Jin G: Ulinastatin protects rats from sepsisinduced acute lung injury by suppressing the JAK-STAT3 pathway. J Cell Biochem 120: 2554-2559, 2018

40. Chow F, Ozols E, Nikolic-Paterson DJ, Atkins RC and Tesch GH: Macrophages in mouse type 2 diabetic nephropathy: Correlation with diabetic state and progressive renal injury. Kidney Int 65 : 116-128, 2004.
41. Zhou J, Li Z, Wu T, Zhao Q and Cao Y: LncGBP9/miR-34a axis drives macrophages toward a phenotype conducive for spinal cord injury repair via STAT1/STAT6 and SOCS3. J Neuroinflammation 17: 020-01805, 2020.

42. Lee JW, Chun W, Lee HJ, Min JH, Kim SM, Seo JY, Ahn KS and Oh SR: The role of macrophages in the development of acute and chronic inflammatory lung diseases. Cells 10: 897, 2021.

43. Gordon S and Taylor PR: Monocyte and macrophage heterogeneity. Nat Rev Immunol 5: 953-964, 2005.

44. Gordon S: Alternative activation of macrophages. Nat Rev Immunol 3: 23-35, 2003.

45. Ji L, Chen Y, Wang H, Zhang W, He L, Wu J and Liu Y: Overexpression of Sirt6 promotes M2 macrophage transformation, alleviating renal injury in diabetic nephropathy. Int J Oncol 55: 103-115, 2019.

This work is licensed under a Creative Commons Attribution-NonCommercial-NoDerivatives 4.0 International (CC BY-NC-ND 4.0) License. 